\title{
ON VARIETIES OF GROUPS WITHOUT POSITIVE LAWS
}

\author{
P. Kozhevnikov ${ }^{1}$ and O. Macedońska ${ }^{2}$ \\ ${ }^{1}$ Department of Mechanics and Mathematics, Moscow State \\ University, Moscow 117234, Russia \\ ${ }^{2}$ Institute of Mathematics, Silesian University of Technology, \\ Gliwice 44-100, Poland
}

\begin{abstract}
We give negative answers to three questions concerning positive laws and $\mathfrak{A}_{p} \mathfrak{A}$ varieties.
\end{abstract}

Let $\mathfrak{A}$ denote the variety of all abelian groups and $\mathfrak{A}_{p}$ - the variety of all abelian groups of exponent $p$. By $F$ we denote a free group and by $V-$ a verbal subgroup in $F$.

We write $G \supseteq \mathcal{F}$ to say that $G$ contains a free nonabelian subsemigroup. A variety generated by $G$ is denoted by $\operatorname{var}(G)$.

A law $u\left(x_{1}, \ldots, x_{n}\right)=u^{\prime}\left(x_{1}, \ldots, x_{n}\right)$ is called positive if $u, u^{\prime}$ are positive words, i.e. words written without inverses of variables. Each positive law implies a binary positive law. If a group $G$ satisfies a positive law, then $\operatorname{var}(G)$ has a basis of positive laws [3]. By Zorn Lemma there exist minimal varieties without positive laws, so called just not p.l. varieties. It follows from [1], that varieties $\mathfrak{A}_{p} \mathfrak{A}$ for prime $p$ are just not p.l. varieties.

For each finitely generated relatively free group $G$ the following questions either have a positive answer or none.

Question 1. Let $G$ contain a free nonabelian subsemigroup. Does $\operatorname{var}(G)$ contain $\mathfrak{A}_{p} \mathfrak{A}$ for some $p$ ?

Question 2. Let $G$ contain a free nonabelian subsemigroup. Does $G / G^{\prime \prime}$ also contain a free nonabelian subsemigroup?

\footnotetext{
${ }^{1}$ e-mail: kozhevn@mccme.ru

${ }^{2}$ e-mail: olga@zeus.polsl.gliwice.pl
} 
Lemma. For a finitely generated relatively free group $G$ the above Questions are equivalent.

Proof. We get the required equivalence by proving the following implications:

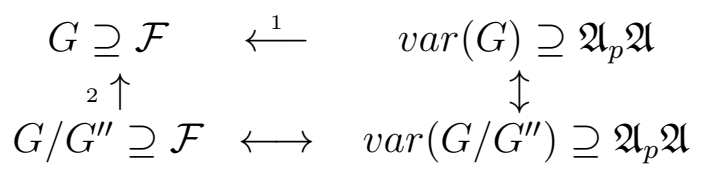

In implication 1 we have that a verbal subgroup $V \subseteq F$, corresponding to $\operatorname{var}(G)$ satisfies $V \subseteq F^{\prime \prime}\left(F^{\prime}\right)^{p}$ for some $p$. By result of Mal'cev [4], $F / F^{\prime \prime}\left(F^{\prime}\right)^{p}$ contains a free nonabelian subsemigroup and hence the relatively free group $G$ also contains a free nonabelian subsemigroup, which proves the implication. Implication 2 is clear.

Consider now the horisontal equivalence. By result of Rosenblatt [6] for finitely generated soluble groups: $G / G^{\prime \prime}$ either contains a free nonabelian subsemigroup or is nilpotent-by-finite. By result of Groves [1] for finitely generated soluble groups: either $\operatorname{var}\left(G / G^{\prime \prime}\right)=\mathfrak{A}_{p} \mathfrak{A}$ for some $p$, or $G / G^{\prime \prime}$ is nilpotent-by-finite. These imply the horisontal equivalence.

The vertical equivalence follows, because for a verbal subgroup $V$, the inclusion $V \subseteq F^{\prime \prime}\left(F^{\prime}\right)^{p}$ is equivalent to $F^{\prime \prime} V \subseteq F^{\prime \prime}\left(F^{\prime}\right)^{p}$.

Since our Questions concern the implications inverse to 1, 2, their equivalence follows.

Corollary. A negative answer to the above Questions implies the existence of a just not p.l. variety different from $\mathfrak{A}_{p} \mathfrak{A}$ for any $p$. This solves the problem posed in ([7] 19.2): whether $\mathfrak{A}_{p} \mathfrak{A}$ are the only just not p.l. varieties?

Proof. Since in a relatively free group $G$ any relation on free generators is a law, we have that $G$ contains a free nonabelian subsemigroup if and only if $G$ does not satisfy a positive law. So the negative answer to Question 1 means that there exists $G$ such that $\operatorname{var}(G)$ does not satisfy positive laws and does not contain $\mathfrak{A}_{p} \mathfrak{A}$ for any $p$. This implies that $\operatorname{var}(G)$ contains a just not p.l. variety different from any of $\mathfrak{A}_{p} \mathfrak{A}$.

To show that the questions have negative answer, we consider the relatively free group $G$ with two or more free generators, defining a pseudoabelian variety (without non-abelian metabelian subgroups) which is studied in Chapter 9 of [5]. Let

$$
w(x, y)=[x, y] v(x, y)^{n}[x, y]^{e_{1}} v(x, y)^{n+1} \ldots[x, y]^{e_{h-1}} v(x, y)^{n+h-1},
$$


where $v(x, y)=\left[\left[x^{d}, y^{d}\right]^{d},\left[y^{d}, x^{-d}\right] d\right], h \equiv 1(\bmod 10), e_{10 k+1}=e_{10 k+2}=$ $e_{10 k+3}=e_{10 k+5}=e_{10 k+6}=1, e_{10 k+4}=e_{10 k+7}=e_{10 k+8}=e_{10 k+9}=e_{10 k+10}=$ $-1, k=0,1, \ldots,(h-1) / 10$ and $d, n, h$ are sufficiently large natural numbers chosen with respect to the restrictions that are introduced in Chapter 7 of [5]. Note that $e_{1}+\cdots+e_{h-1}=0 . G$ is defined by the law $w(x, y)=1$.

Theorem. The group $G$ defined above contains a free non-abelian subsemigroup, while var $(G)$ does not contain $\mathfrak{A}_{p} \mathfrak{A}$ for any $p$.

Proof Assume that $\operatorname{var}(G)$ contains $\mathfrak{A}_{p} \mathfrak{A}$ for some $p$, then $G \cong F / V$ and $V \subseteq F^{\prime \prime}\left(F^{\prime}\right)^{p}$. This implies $G / G^{\prime \prime}\left(G^{\prime}\right)^{p} \cong F / F^{\prime \prime}\left(F^{\prime}\right)^{p}$, which is not true, because every metabelian group in $\operatorname{var}(G)$ is abelian.

To show that $G$ contains a free non-abelian subsemigroup we use the technique of graded diagrams developed in [5]. All references below concern this book. Let $a, b$ be two distinct free generators in $G$. We are going to prove that the subsemigroup generated by $a$ and $b$ is a free semigroup. Suppose to the contrary that an equality $u(a, b)=u^{\prime}(a, b)$ holds in $G$, for some distinct positive words $u, u^{\prime}$. Without loss of generality we can assume that $u$ and $u^{\prime}$ have distinct leading and ending letters; thus we can assume that the word $u^{-1} u^{\prime}$ is a cyclically reduced non-empty word (one of the words $u, u^{\prime}$ could be an empty word).

Now for the group $G$, defined by the word $w$ we consider a reduced diagram $\Delta$ of the equality $u^{-1} u^{\prime}=1$. The contour of $\Delta$ is presented in the form $p q$, where the section $p$ has the label $\varphi(p)=u^{-1}$ and the section $q$ has the label $\varphi(q)=u^{\prime}$. Since the equality $u^{-1} u^{\prime}=1$ does not hold in a free group, the rank of $\Delta$ is greater than 0 (see $\S 11$ ).

By Theorem 22.2, in $\Delta$ there is an $\mathcal{R}$-cell $\Pi$ and a subdiagram $\Gamma$ of rank 0 satisfying the following conditions: 1$) \Gamma$ is a contiguity subdiagram of a long section $t$ of the contour of $\Pi$ to one of the sections $p$ or $q$; 2) $\Gamma$-contiguity degree of $t$ to $p$ (or $q$ ) is not less than $\varepsilon$ (see Chapter $7, \S 20$ ). This implies (by reasoning similar to that in Theorem 19.1) that one of the labels $\varphi(p)$, $\varphi(q)$ has a common subword of length $|A|^{[\varepsilon n]}$ with the section $t$, where $A$ is a period corresponding to the cell $\Pi$. In particular, one of the words $\varphi(p)$, $\varphi(q)$ contains either the word $A$ or the word $A^{-1}$ as a subword. Since $\varphi(p)$ is a negative (i.e. containing only negative powers of $a$ and $b$ ) word and $\varphi(q)$ is a positive word, we see that the word $A$ is either positive or negative. From the definition of periods (see $\S 29.3$ ) it follows that $A^{f}$ for some integer $f$ $(f \neq 0$ by Lemma 30.3) is conjugate in $G$ to a word $v(X, Y)$ for some words $X, Y$ and the word $v$ defined above. The word $v$ is a commutator word (i.e. $v(X, Y)$ belongs to the commutator subgroup of a free group with free 
generators $X, Y)$, hence $A^{f} \in G^{\prime}$. The variety $\operatorname{var}(G)$ is defined by the law $w(x, y)=1$, where $w$ is a commutator word; hence, $\mathfrak{A} \subseteq \operatorname{var}(G)$. Therefore $A^{f}$ is a commutator word (i.e. $A^{f}$ belongs to the commutator subgroup $F^{\prime}$ of the free group $F=F(a, b)$ freely generated by $a$ and $b)$. Since the factorgroup $F / F^{\prime}$ has no torsion and $f \neq 0$, the word $A$ belongs to $F^{\prime}$. This means that the number of occurrences of the letter $a$ in the word $A$ is equal to the number of occurrences of the letter $a^{-1}$, and the same is true for the letters $b$ and $b^{-1}$. Hence the word $A$ is neither positive nor negative. We get the contradiction which ends the proof.

Corollary. There exists continuously many just not p.l. varieties.

Proof. It follows from the results obtained in [2], that there exists a continuous set of infinitely based pseudo-abelian varieties with pairwise intersections equal to $\mathfrak{A}$. Since the Theorem holds if we replace $\operatorname{var}(G)$ by any of these varieties, the statement follows.

\section{References}

[1] Groves, J.R.J. Varieties of soluble groups and a dichotomy of P.Hall. Bull. Austral. Math. Soc. 1971, 5, 391-410.

[2] Kozhevnikov, P.A. On group varieties of large odd exponent. Deposited in VINITI 05.06.2000, 1612-00, 26 pp.

[3] Lewin, J.; Lewin, T. Semigroup laws in varieties of soluble groups. Proc. Camb. Phil. Soc. 1969, 65, 1-9.

[4] Mal'cev, A.I. Nilpotent semigroups. Uchen. Zap. Ivanovsk. Ped. Inst. 1953, 4, 107-111.

[5] Ol'shanskii, A.Yu. Geometry of defining relations in groups; Mathematics and its applications (Soviet Series), 70; Kluwer Academic Publishers: Dordrecht, 1991.

[6] Rosenblatt, J.M. Invariant measures and growth conditions. Trans. Am. Math. Soc. 1974, 193, 33-53.

[7] L. N. Shevrin, M. V. Volkov, Identities of semigroups (in Russian), Izv. Vyssh. Uchebn. Zaved. Mat. 11 (1985), 3-47. English translation: Soviet Math. (Iz. VUZ) 29 (1985), no. 11, 1-64. 